\title{
RELACIÓN ENTRE EL BIENESTAR EMOCIONAL Y EL PROCESO DE COMU- NICACIÓN DE MALAS NOTICIAS Y TOMA DE DECISIONES EN PERSONAS DIAGNOSTICADAS DE TUMOR CEREBRAL MALIGNO
}

Tipo de trabajo: Tesis doctoral

Autor: José Luis Díaz Cordobés

Directores: Dra. María Pilar Barreto Martín / Dr. Juan Antonio Barcia Albacar

Centro: Departamento de Personalidad, Evaluación y tratamientos psicológicos. Facultad de Psicología. Universidad de Valencia / Servicio de Neurocirugía. Hospital General Universitario de Valencia.

Fecha de lectura: 14 Marzo de 2011.

E-mail: jldiazcordobes@yahoo.es

\section{Objetivos:}

- Estudiar la evolución del estado emocional de los pacientes diagnosticados de Glioblastoma Multiforme desde el momento del diagnóstico hasta el momento del fallecimiento.

- Describir las características sociodemográficas, clínicas y psicológicas de los pacientes incluidos en el estudio.

- Explorar la relación diferencial entre variables clínicas, bienestar del paciente y variables relacionadas con la provisión de información (grado de información proporcionada al paciente y el proceso de toma de decisiones terapéuticas).

- Establecer el peso relativo de estas variables (información, toma de decisiones y datos clínicos) en la predicción del Bienestar Emocional en una muestra de pacientes diagnosticados de tumor cerebral.

Sujetos: 58 pacientes con tumor cerebral maligno atendidos en el Servicio de Neurocirugía del Hospital General Universitario de Valencia desde enero 2005 hasta diciembre 2007. Todos los pacientes se encontraban conscientes y orientados temporoespacialmente en el momento del diagnóstico.

Metodología: Las variables consideradas fueron las siguientes: variables sociodemográficas (edad, género, nivel de estudios), variables clínicas (localización del tumor y línea de tratamiento), variables de información (deseo de conocer, nivel de información, comprensión de la información, aspectos que no le hubiesen gustado conocer, oportunidad de preguntar y resolver dudas, atención emocional y satisfacción con la información recibida. Las variables relacionadas con la información fueron administradas tanto a pacientes como a médicos responsables), malestar emocional (ansiedad y depresión -HADs-) y malestar global (escala análogo-visual 0-10). Los momentos de evaluación fueron los siguientes: momento de ingreso, inmediatamente después de la información del diagnostico y proceso de toma de decisiones, en el momento del alta hospitalaria, un mes después del alta y 6 meses después. En los momentos en los que el paciente se encontraba en domicilio se evaluó el malestar global a través de la escala 0-10 vía telefónica.

\section{Conclusiones:}

- Los pacientes diagnosticados de GBM presentan mayores niveles de ansiedad que de depresión.

- El momento de mayor vulnerabilidad emocional en relación a la ansiedad, es cuando se confirma el diagnóstico y se decide el tratamiento.

- Desde el diagnóstico hasta el momento del éxitus, los pacientes consideran 
que prácticamente la mitad del tiempo están mal o muy mal.

- Los niveles de ansiedad están relacionados con el proceso de información y toma de decisiones hasta un mes después del alta hospitalaria.

- Los pacientes que reciben información excesiva o innecesaria a sus necesidades presentan mayores niveles de ansiedad; no se tienen en cuenta sus emociones presentan mayor malestar emocional, se sienten menos satisfechos con el proceso tienen mayor ansiedad y su familia intenta ocultar información a pesar de que el propio enfermo quiere conocerla presentan mayores niveles de ansiedad.
- Los pacientes que presentan una mayor satisfacción con la atención recibida son aquellos que desean estar informados acerca su enfermedad, que han comprendido mejor la información, que consideran que han recibido información suficiente, que no han recibido información que no necesitan y que consideran que durante el proceso el equipo se ha hecho cargo de sus emociones.

- Los médicos consideran que deberían dar más cantidad de información de la que en realidad los pacientes necesitan, pero hay determinados aspectos que los pacientes necesitan abordar y los médicos no consideran oportuno hacerlo. 